\title{
Human Amnion-Derived Mesenchymal Stem Cells Protect Human Bone Marrow Mesenchymal Stem Cells against Oxidative Stress-Mediated Dysfunction via ERK1/2 MAPK Signaling
}

\author{
Yuli Wang ${ }^{1,2}$, Junchi Ma ${ }^{1,2}$, Yifei Du', Jing Miao', and Ning Chen ${ }^{1, *}$
}

\begin{abstract}
Epidemiological evidence suggests that bone is especially sensitive to oxidative stress, causing bone loss in the elderly. Previous studies indicated that human amnionderived mesenchymal stem cells (HAMSCs), obtained from human amniotic membranes, exerted osteoprotective effects in vivo. However, the potential of HAMSCs as seed cells against oxidative stress-mediated dysfunction is unknown. In this study, we systemically investigated their antioxidative and osteogenic effects in vitro. Here, we demonstrated that HAMSCs significantly promoted the proliferation and osteoblastic differentiation of $\mathrm{H}_{2} \mathrm{O}_{2}$-induced human bone marrow mesenchymal stem cells (HBMSCs), and down-regulated the reactive oxygen species (ROS) level. Further, our results suggest that activation of the ERK1/2 MAPK signal transduction pathway is essential for both HAMSCs-mediated osteogenic and protective effects against oxidative stress-induced dysfunction in HBMSCs. U0126, a highly selective inhibitor of extracellular ERK1/2 MAPK signaling, significantly suppressed the antioxidative and osteogenic effects in HAMSCs. In conclusion, by modulating HBMSCs, HAMSCs show a strong potential in treating oxidative stress- mediated bone deficiency.
\end{abstract}

\section{INTRODUCTION}

Bone deficiency is an emerging medical and socioeconomic challenge among patients requiring dental implants. It not only impairs the long-term stability of dental implants, but also the osseointegration of implants and bone (Maire, 1997). Further,

\footnotetext{
${ }^{1}$ Jiangsu Key Laboratory of Oral Diseases, Nanjing Medical University, Nanjing 210029, Jiangsu, People's Republic of China, ${ }^{2}$ These authors contributed equally to this work.

*Correspondence: njcn@njmu.edu.cn
}

Received 3 June, 2015; revised 17 November, 2015; accepted 23 November, 2015; published online 7 January, 2016

Keywords: ERK1/2 MAPK signal transduction pathway, human amnion mesenchymal stem cells (HAMSCs), human bone marrow mesenchymal stem cells (HBMSCs), oxidative stress, reactive oxygen species (ROS) bone deficiency significantly affects patients' appearance, decreases the stability of dentures, and increases the difficulty of restoring oral function (Hertz, 1956). Increasing evidence suggests a biochemical link between increased oxidative stress and bone deficiency (Basu et al., 2001).Increase in oxidative stress and decreased capacity for antioxidant defense (Muthusami et al., 2005) results in decreased bone mineral density (BMD) in postmenopausal women (Ozgocmen et al., 2007; Sendur et al., 2009; Witko-Sarsat et al., 1996). Excessive production of reactive oxygen species (ROS), due to oxidative stress triggers bone resorption with a direct effect on osteoclastinduced superoxide generation and bone deficiency (Sontakke and Tare, 2002; Yang et al., 2001). The results indicate that oxidative stress is a crucial factor underlying bone deficiency (Sendur et al., 2009), inhibits osteoblastic differentiation (Fatokun et al., 2008; Mody et al., 2001) and increases osteoclast differentiation and function (Sontakke and Tare, 2002).

For instance, antioxidants such as ophiopogonin D (Huang et al., 2015), resveratrol (Haigis and Sinclair, 2010) and flavones (Zeng et al., 2014), have been used to treat oxidative stressinduced bone deficiency. The disadvantages include remarkable individual differences and limited availability. Tissue engineering using appropriate seed cells has shown great potential in the treatment of bone deficiency (Ito et al., 2006; Kim et al., 2009; Wang et al., 2009; Zhao et al., 2009). Human amnionderived mesenchymal stem cells (HAMSCs), obtained from human amniotic membrane (AM) are a readily available and highly abundant tissue (Bourne, 1960), with substantial benefits as seed cells. Their low anti-inflammatory properties and fewer ethical concerns compared with other sources of stem cells are clear advantages (Leyva-Leyva et al., 2013). A previous study has confirmed that HAMSCs provide an environment conducive to osteogenic differentiation in human bone marrow mesenchymal stem cells (HBMSCs)(Wang et al., 2014). We, therefore, hypothesized that HAMSCs play a role in oxidative stressinduced bone deficiency. In this study, we used a Transwell coculture system to investigate the in vitro effects of HAMSCs on osteogenic differentiation in $\mathrm{H}_{2} \mathrm{O}_{2}$-induced HBMSCs. Interestingly, we found that HAMSCs promoted proliferation, activated alkaline phosphatase activity (ALP), enhanced the expression of osteogenic marker proteins and stimulated mineralized matrix deposition. We showed that HAMSCs were not only capable of promoting osteogenic differentiation, but also reversing 
the oxidative stress-induced suppression of osteogenic differentiation.

Bone formation, associated with the differentiation of mesenchymal stem cells, is regulated by multiple signaling pathways, including Wnt- $\beta$-Catenin, MAPK, Hedgehog and NF-KB (Galli et al., 2010; Hammond and Schulte-Merker, 2009; Krum et al., 2010; Schindeler and Little, 2006; Simmons et al., 2003). Signal transduction pathways involved in oxidative stress-induced inhibition of osteogenic differentiation consist of MAPK, Akt/mTOR/4EBP1, p53 and NF-kB (Martindale and Holbrook, 2002; Zeng et al., 2014). Previous studies revealed that ERK1/2 MAPK signaling regulates the differentiation and proliferation of HBMSCs (Salasznyk et al., 2004; Xia et al., 1995; Zhang and Liu, 2002). Further, the transcriptional activity of Runt-related transcription factor 2 (Runx2) is activated by ERK1/2 MAPK signaling (Franceschi et al., 2003; Xiao et al., 2002). The phosphorylation of Runx2 is essential for subsequent bone formation and osteoblast differentiation (Franceschi and Xiao, 2003; Salasznyk et al., 2004; Zhang and Liu, 2002). To clarify the underlying mechanisms, we further investigated the role of HAMSCs on ERK1/2 MAPK signaling pathway and Runx2 in $\mathrm{H}_{2} \mathrm{O}_{2}$-induced HBMSCs.

\section{MATERIALS AND METHODS}

\section{Chemicals and reagents}

Fetal bovine serum (FBS), $\alpha$-minimum essential medium $(\alpha M E M)$, trypsin-EDTA, phosphate-buffered saline (PBS) and penicillin G-streptomycin sulfate were purchased from Gibcoß Life Technologies. U0126 (ERK1/2 inhibitor), 2,7-dichlorodihydrofluoresceindiacetate (DCFH-DA), $\beta$-glycerophosphate, ascorbic acid, and dexamethasone were purchased from Sigma-Aldrich (USA). Transwells (6-Well Millicell Hanging Cell Culture Inserts, $0.4 \mu \mathrm{m}, \mathrm{PET}$ ) and 6-well culture plates were purchased from Millipore ${ }^{\circ}$ (USA). The rabbit anti-phospho-Runx2 (Ser451) was purchased from BIOSS Bioscience (China).The Cell-Light ${ }^{\mathrm{TM}}$ EdU Apolloß488 In Vitro Imaging Kit was purchased from RiboBio (China). The goat anti-rabbit IgG, phospho-p44/42 MAPK rabbit $\mathrm{mAb}(\mathrm{p}-\mathrm{ERK} 1 / 2)$, p44/42 MAPK (ERK1/2) rabbit mAb, and Runx2 (D1L7F) rabbit mAb were purchased from Cell Signaling Technology (USA).The protein assay kit, RIPA buffer, and DAPI were purchased from Beyotime (China). The alkaline phosphatase (ALP) assay kit and bicinchoninic acid (BCA) assay kit were purchased from the Jiancheng Corp (China).Other reagents used were of the highest commercial grade available.

\section{Cell culture}

The HBMSCs were obtained from the American Type Culture Collection (ATCC, USA). HAMSCs were collected from abandoned amniotic membrane samples obtained within $24 \mathrm{~h}$ using the pancreatin/collagenase digestion method as previously described (Zhang et al., 2011). Both cells were cultured in $\alpha \mathrm{MEM}$ supplemented with $10 \% \mathrm{FBS}, 100 \mathrm{U} / \mathrm{L}$ penicillin and 100 $\mathrm{mg} / \mathrm{L}$ streptomycin in a humidified atmosphere of $5 \% \mathrm{CO}_{2}$ at $37^{\circ} \mathrm{C}$. Cells from passages $3-5$ were used in this study and the culture medium was changed every 3 days.

\section{The co-culture system}

A Transwell co-culture system was used to investigate the effects of HAMSCs on proliferation and osteogenic differentiation in $\mathrm{H}_{2} \mathrm{O}_{2}$-induced HBMSCs. HBMSCs were seeded at an initial density of $5 \times 10^{4}$ cells $/ \mathrm{cm}^{2}$ in 6 -well culture plates. Transwells were placed in other 6 -well culture plates and seeded at in- creasing HBMSCs:HAMSCs ratios $\left(5 \times 10^{4}\right.$ cells/transwell, $10 \times$ $10^{4}$ cells/transwell and $15 \times 10^{4}$ cells/transwell). After approximately $24 \mathrm{~h}, \mathrm{HBMSC}$ were incubated with serum-free medium or $200 \mu \mathrm{M} \mathrm{H}_{2} \mathrm{O}_{2}$ for $12 \mathrm{~h}$ (Bai et al., 2004; Liu et al., 2004) to induce oxidative injury as previously described. After washing with PBS, transwells containing HAMSCs were transferred into the corresponding wells of the 6-well culture plate containing HBMSCs to create the HAMSC/HBMSCs Transwell co-culture system. HBMSCs in wells with transwells served as the treatment groups, while HBMSCs without transwells were designated as the control groups.

\section{Analysis of cellular proliferation}

Cellular proliferation was measured by flow cytometry at 3, 5 and 7 days and via immunofluorescence staining of 5-ethynyl2'-deoxyuridine (EdU) at 5 days. Briefly, after starvation in serum-free medium or $200 \mu \mathrm{M} \mathrm{H}_{2} \mathrm{O}_{2}$ for $12 \mathrm{~h}$, HBMSCs were washed with PBS. Transwells containing HAMSCs were moved into the corresponding wells of the 6-well culture plate containing HBMSCs in routine culture media supplemented with $10 \%$ FBS. At 3, 5 and 7 days, Transwells containing HAMSCs were removed and HBMSCs were harvested. After fixing with $75 \%$ ice-cold ethanol at $4^{\circ} \mathrm{C}$ in the dark (Wang et al., 2013), cell cycle fractions (G0, G1, S, and G2 M phases) were determined by flow cytometry. A Cell-Light ${ }^{\mathrm{TM}}$ EdU Apolloß488 In Vitro Imaging Kit was used to investigate the EdU levels in each group at 5 days. Briefly, $100 \mu \mathrm{l} \mathrm{EdU} \mathrm{medium}(50 \mu \mathrm{M})$ was added to each well and incubated for $2 \mathrm{~h}$. The EdU medium was removed and HBMSCs were washed twice with PBS and fixed with $75 \%$ ethanol. After lysing with Triton X-100 (0.5\%) for 15 min, staining solution was added to each well. After incubating for $30 \mathrm{~min}$, EdU levels were examined by immunofluorescence staining and the area of stained EdU-positive nodules relative to the total culture surface was measured by Image-pro Plus analysis (IPP). DAPI was used to stain the cell nuclei.

\section{Assessment of ROS production}

The level of ROS induced by $\mathrm{H}_{2} \mathrm{O}_{2}$ in HBMSCs was measured using DCFH-DA as a fluorescent probe. After co-culturing with HAMSCs for $48 \mathrm{~h}$, transwells containing HAMSCs were removed and HBMSCs were washed twice with PBS, incubated with DCFH-DA (10 mM) for $30 \mathrm{~min}$ at $37^{\circ} \mathrm{C}$ and again washed three times with PBS. Macrographs of DCFDA fluorescence were immediately recorded and the area of stained ROSpositive nodules relative to the total culture surface was measured by Image-pro Plus analysis (IPP). The intensity of 2',7'dichlorofluorescin (DCF) fluorescence was determined by flow cytometry.

\section{In vitro osteogenic differentiation}

After incubating in serum-free medium or $200 \mu \mathrm{M} \mathrm{H} \mathrm{H}_{2} \mathrm{O}_{2}$ for $12 \mathrm{~h}, \mathrm{HBMSC}$ were washed with PBS. Transwells containing HAMSCs were moved into the corresponding wells of the 6 -well culture plate containing HBMSCs. Both cells were cultured in osteogenic medium (OS) containing $10 \mathrm{mM} \beta$ glycerophosphate, $100 \mathrm{nM}$ ascorbic acid, and $100 \mathrm{nM}$ dexamethasone. HBMSCs co-cultured with HAMSCs were then analyzed for ALP activity and mineralized matrix formation followed by, Western blotting analysis and flow cytometry. U0126, a highly selective inhibitor of ERK $1 / 2$ signaling, was prepared in DMSO and used in the signaling inhibition assay at the concentration of $20 \mathrm{mM}$ as previously described (Chen et al., 2012). To eliminate the influence of U0126 on HAMSCs, $\mathrm{H}_{2} \mathrm{O}_{2}$-induced HBMSCs were treated with U0126 for $24 \mathrm{~h}$ after incubating in $200 \mu \mathrm{M} \mathrm{H}_{2} \mathrm{O}_{2}$ before co-culture with HAMSCs. 
$\boldsymbol{A}$

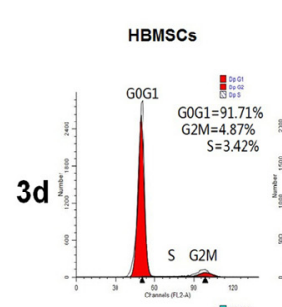

5d

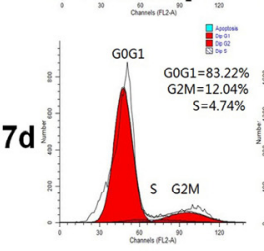
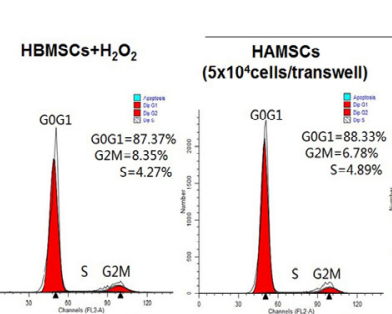

HBMSCs $+\mathrm{H}_{2} \mathrm{O}_{2}+\mathrm{HAMSCs}$
HAMSCs HAMSCs (10x104cells/transwell)
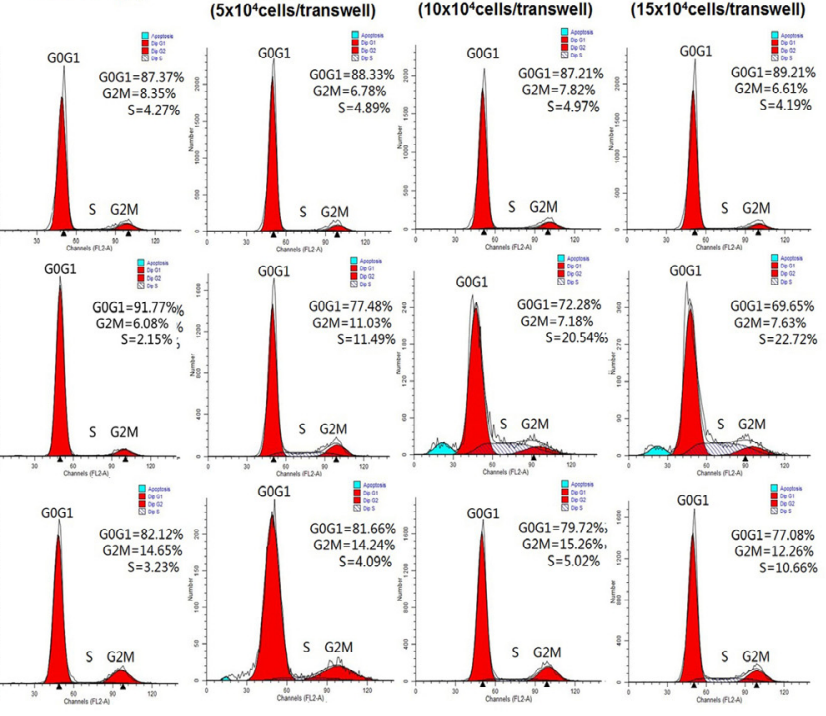

B

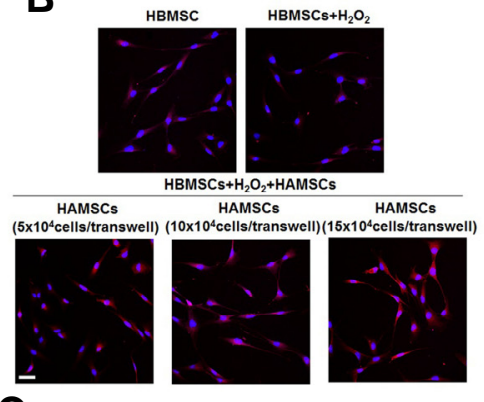

C

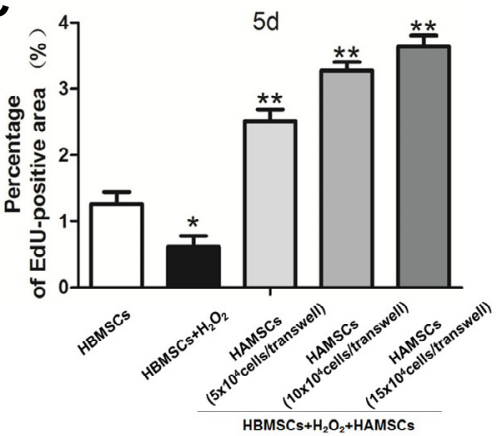

Fig. 1. The effect of HAMSCs on $\mathrm{H}_{2} \mathrm{O}_{2}$-induced HBMSCs proliferation was determined by flow cytometry and immunofluorescence staining of 5-ethynyl-2'-deoxyuridine (EdU), (A) Cell cycle fractions (G0, G1, S, and G2 M phases) at 3, 5 and 7 days. (B) Immunofluorescence staining of EdU at 5 days. (C) The area of stained EdU-positive nodules relative to the total culture surface at 5 dats was measured by Image-pro Plus analysis (IPP). Scale bar, $100 \mu \mathrm{m} ;{ }^{*} \mathrm{P}<0.05$ and ${ }^{* *} \mathrm{P}<0.01$ in contrast to the group treated with $\mathrm{H}_{2} \mathrm{O}_{2}$ alone.

Assessment of ALP activity and mineralized matrix formation

Fourteen days after co-culturing, Transwells containing HAMSCs were removed. HBMSCs in each group were then washed twice with PBS and lysed with Triton X-100 (0.5\%) for $15 \mathrm{~min}$. A bicinchoninic acid (BCA) assay kit was used to determine the protein concentration. ALP activity was performed using the alkaline phosphatase (ALP) assay kit (Jiancheng Corp, China) according to the manufacturer's instructions and estimated based on the absorbance at $405 \mathrm{~nm}$ as previously described (Ozeki et al., 2008; Schneider et al., 1999; Wang et al., 2012). The enzyme activity was expressed as micromoles of reaction product per minute per total protein.

HBMSCs co-cultured with HAMSCs for 21 days were stained with Alizarin red $S$ to assess mineralized matrix deposition for bone nodule formation (Reinholz et al., 2000; Wang et al., 2012). Cells were washed twice with PBS, fixed with $75 \%$ dehydrated alcohol and then stained with $40 \mathrm{mM}$ alizarin red $\mathrm{S}$ (pH 4.4) for $10 \mathrm{~min}$ at room temperature. After rinsing with PBS, nodules were visualized using an inverted microscope and the area of mineralized nodules was measured by Image-pro Plus analysis (IPP). Ten images were captured for each group, and the mean percentage was calculated.

Assessment of osteoblast (OB) -related protein

At the end of the 14-day co-culture, Transwells containing HAMSCs were removed and HBMSCs in each group were lysed in RIPA buffer containing $1 \mathrm{mM}$ phenylmethane sulfonylfluoride according to the manufacturer's instructions. The total protein concentration was determined using a bicinchoninic acid (BCA) assay kit. Protein lysates $(20 \mu \mathrm{g})$ were separated by sodium dodecyl sulfate-polyacrylamide gel electrophoresis (SDS-PAGE) and then transferred onto $0.22 \mu \mathrm{m}$ polyvinylidene difluoride membranes (Millipore, USA). After blocking, membranes were incubated overnight at $4^{\circ} \mathrm{C}$ with specific antibodies for the detection of p-ERK1/2(1:500), ERK1/2(1:500), and Runx2(1:1,000). After three washes with PBST $(0.5 \%$ Tween 20 in PBS), the membranes were incubated with the relevant secondary antibodies $(1: 1,000)$ for $1 \mathrm{~h}$ at $37^{\circ} \mathrm{C}$, washed and visualized with an ECL detection kit (Amersham Pharmacia, USA). The $\beta$-actin (1:500) served as an internal control.

The level of phospho-Runx 2 was measured by flow cytometric analysis. Fourteen days after co-culturing, Transwells containing HAMSC.s were remnved HBMSCs in each group were fixed with $1 \%$ paraformaldehyde for $10 \mathrm{~min}$ at $37^{\circ} \mathrm{C}$ and then permeablized with Triton X-100 (0.5\%) for 30 min on ice. After washing with PBS, cells were stained with anti-phospho-Runx2 (Ser451) antibody (1:200) for $20 \mathrm{~min}$ at $37^{\circ} \mathrm{C}$ in the dark. The cells were incubated with the relevant secondary antibodies and analyzed on a flow cytometer after washing with PBS.

\section{Statistical analysis}

All data were expressed as mean \pm S.D. from at least three independent experiments, each performed in quintuplicate. Data were analyzed using one-way analysis of variance (ANOVA). $P$-values $<0.05$ were considered statistically significant.

\section{RESULTS}

\section{HAMSCs promoted $\mathrm{H}_{2} \mathrm{O}_{2}$-induced $\mathrm{HBMSC}$ s proliferation}

Flow cytometry and immunofluorescence staining of 5-ethynyl2'-deoxyuridine (EdU) were used to measure the proliferation of $\mathrm{H}_{2} \mathrm{O}_{2}$-induced HBMSCs seeded in the transwell co-culture system. Cell cycle fractions (G0, G1, S, and G2M phases) were determined by flow cytometry at 3,5 and 7 days. At 3 and 7 
$\boldsymbol{A}$

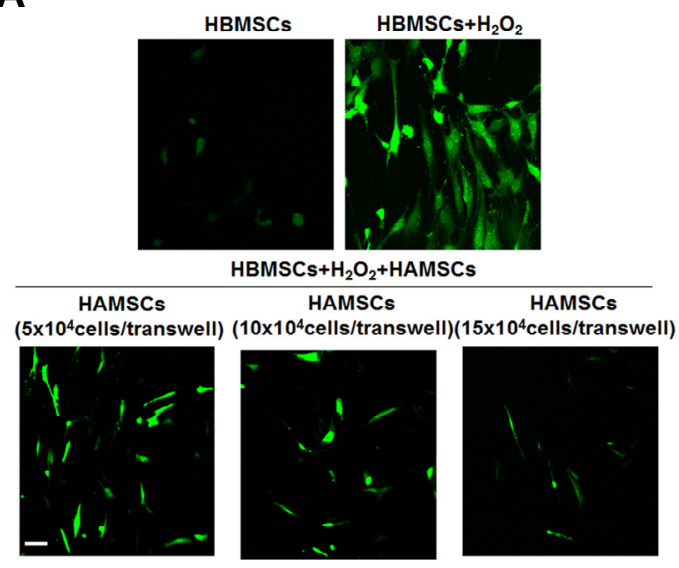

B

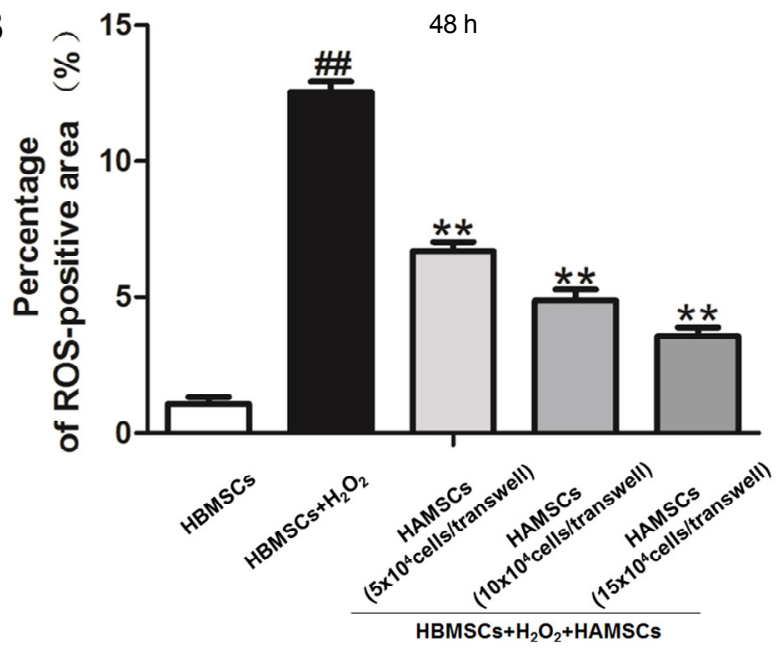

C

HBMSCs

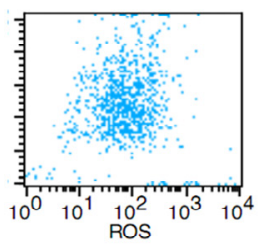

$\frac{X \text { Mean }}{165.55}$
$\mathrm{HBMSCs}+\mathrm{H}_{2} \mathrm{O}_{2}$

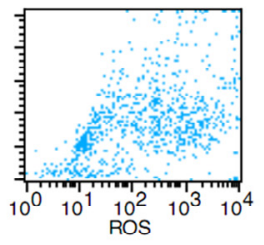

$\frac{\mathrm{X} \text { Mean }}{1036.07}$
HAMSCs (5x104cells/transwell)

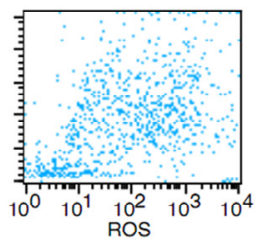

$\frac{\mathrm{X} \text { Mean }}{591.77}$
$\mathrm{HBMSCs}+\mathrm{H}_{2} \mathrm{O}_{2}+\mathrm{HAMSCs}$

$\begin{array}{cc}\text { HAMSCs } & \text { HAMSCs } \\ \left(10 \times 10^{4} \text { cells/transwell }\right) & \left(15 \times 10^{4} \text { cells/transwell }\right)\end{array}$
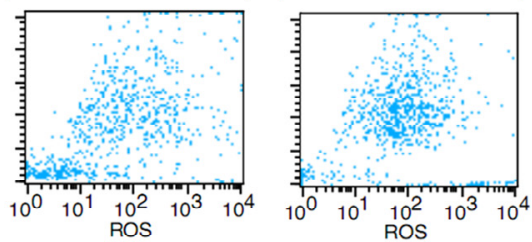

$\frac{X \text { Mean }}{428.93}$

Fig. 2. Flow cytometry and immunofluorescence staining of ROS generation in $\mathrm{H}_{2} \mathrm{O}_{2}$-induced $\mathrm{HBMSCs}$ after co-culturing with or without HAMSCs (A) Macrographs of DCFDA fluorescence were detected by fluorescence microscopy at $48 \mathrm{~h}$. (B) The area of stained ROS-positive nodules relative to the total culture surface at $48 \mathrm{~h}$ was measured by Image-pro Plus analysis (IPP). (C) The intensity of fluorescence was determined by flow cytometry at $48 \mathrm{~h}$. Scale bar, $100 \mu \mathrm{m} ;{ }^{*} \mathrm{P} P 0.01$ vs. the HBMSCs group; ${ }^{*} \mathrm{P}<0.05$ and ${ }^{* *} \mathrm{P}<0.01 \mathrm{compared}$ with the group treated with $\mathrm{H}_{2} \mathrm{O}_{2}$ alone.

days, the S-phase showed no significant difference between each groups. However, the proliferation of HBMSCs was significantly inhibited by $\mathrm{H}_{2} \mathrm{O}_{2}$ and the S-phase checkpoints increased slightly with the HBMSCs:HAMSCs ratio at 5 days (Fig. 1A). Further, immunofluorescence staining of EdU, a cell proliferation marker, revealed a statistically significant increase of EdU-positive HBMSCs nodules with the HBMSCs:HAMSCs ratio in co-culture groups compared with the single-culture groups at 5 days (Figs. $1 \mathrm{~B}$ and $1 \mathrm{C}$ ). Previous study had shown that co-culturing with HAMSCs promoted HBMSCs proliferation in the absence of hydrogen peroxide (Wang et al., 2015).Our results further demonstrated that coculturing with HAMSCs also accelerated $\mathrm{H}_{2} \mathrm{O}_{2}$-induced HBMSCs proliferation.

\section{HAMSCs inhibited ROS generation in $\mathrm{H}_{2} \mathrm{O}_{2}$-induced HBMSCS}

To elucidate whether the beneficial effects of HAMSCs were linked to their antioxidant properties, the ROS generation in $\mathrm{H}_{2} \mathrm{O}_{2}$-induced HBMSCs was measured. Subsequently, macrographs of DCFDA fluorescence were analyzed qualitatively using fluorescence microscopy and the area of stained ROSpositive nodules relative to the total culture surface was meas- ured by Image-pro Plus analysis (IPP). The intensity of fluorescence was determined by flow cytometry. As shown in Figs. $2 \mathrm{~A}, 2 \mathrm{~B}$ and $2 \mathrm{C}, \mathrm{H}_{2} \mathrm{O}_{2}$ significantly increased the ROS generation in other groups. However, when HBMSCs were co-cultured with HAMSCs, ROS generation was suppressed partially compared with the $\mathrm{H}_{2} \mathrm{O}_{2}$-induced single-culture groups and the effect of suppression increased with the HBMSCs: HAMSCs ratio. Our data demonstrated that coculturing with HAMSC inhibited the oxidative status in $\mathrm{H}_{2} \mathrm{O}_{2-}$ induced HBMSCs and the beneficial effects of HAMSCs might be linked to its antioxidant properties.

\section{HAMSCs promoted ALP activity and mineralized matrix formation in $\mathrm{H}_{2} \mathrm{O}_{2}$-induced $\mathrm{HBMSCS}$}

Figure $3 \mathrm{~A}$ shows the proportion of cultured HBMSCs staining positively for extracellular matrix in the presence or absence of $\mathrm{H}_{2} \mathrm{O}_{2}$ after 21 days co-culturing with and without HAMSCs. In contrast to $\mathrm{H}_{2} \mathrm{O}_{2}$-untreated $\mathrm{HBMSCs}, \mathrm{H}_{2} \mathrm{O}_{2}$ significantly inhibited the mineralized matrix formation in other groups. When $\mathrm{H}_{2} \mathrm{O}_{2}$-induced HBMSCs were co-cultured with HAMSCs, higher levels of mineralization were observed after 21 days compared with the $\mathrm{H}_{2} \mathrm{O}_{2}$-induced single-culture groups and the percentage of mineralized nodules increased with the HBMSCs: 
$\boldsymbol{A}$

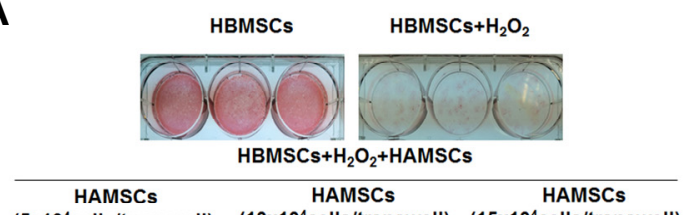

(5x104cells/transwell) $\quad\left(10 \times 10^{4}\right.$ cells/transwell $) \quad\left(15 \times 10^{4}\right.$ cells/transwell $)$
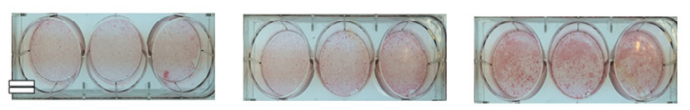

C

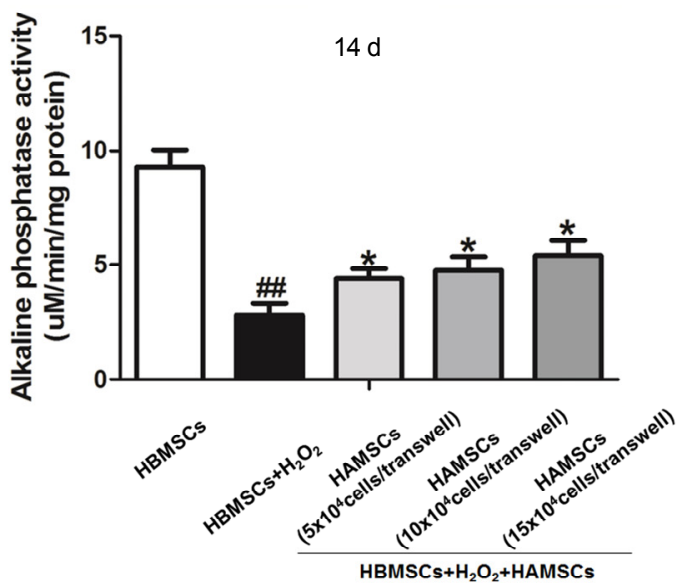

$B$

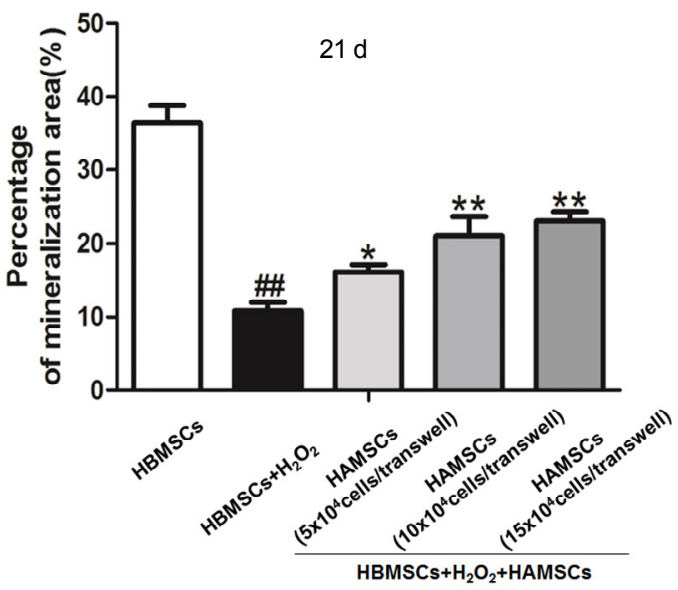

Fig. 3. ALP activity and mineralized matrix deposition in $\mathrm{H}_{2} \mathrm{O}_{2}$-induced HBMSCs cultured with or without HAMSCs. (A) Mineralized matrix deposition was measured at 21 days by Alizarin red S. (B) Micrographs of mineralized matrix deposition were measured by Image-pro Plus analysis (IPP). (C) ALP activity was measured at 14 days using ALP assay kit. Double bar, $12.5 \mathrm{~mm}$; ${ }^{\# \#} \mathrm{P}<0.01$ versus the HBMSCs group; ${ }^{\mathrm{P}}$ $<0.05$ and ${ }^{* *} \mathrm{P}<0.01$ in contrast to the group treated with $\mathrm{H}_{2} \mathrm{O}_{2}$ alone.

HAMSCs ratio (Fig.3B). These observations demonstrated that HAMSCS positively regulated the mineralization of $\mathrm{H}_{2} \mathrm{O}_{2}$ induced HBMSCs in vitro.

ALP activity in HBMSCs co-cultured with and without HAMSCs was measured after 14 days irrespective of $\mathrm{H}_{2} \mathrm{O}_{2}$. Compared with untreated HBMSCs, ALP activity decreased significantly in the $\mathrm{H}_{2} \mathrm{O}_{2}$-induced groups and gradually increased with the HBMSCs:HAMSCs ratio (Fig. $3 \mathrm{C}$ ), indicating the potential effect of HAMSC on osteoblastic differentiation in $\mathrm{H}_{2} \mathrm{O}_{2}$-induced HBMSC

\section{HAMSCs activated ERK1/2 MAPK signaling and Runx2 in $\mathrm{H}_{2} \mathrm{O}_{2}$-induced HBMSCS}

ERK1/2, a member of the MAPK family, regulates the differentiation, mineralization and proliferation of HBMSCs (Salasznyk et al., 2004; Xia et al., 1995; Zhang and Liu, 2002).The transcriptional activity of Runx2 is essential for osteoblastic differentiation and subsequent bone formation. Therefore, we investigated the effect of HAMSCs on ERK1/2 signal and Runx2 activation in $\mathrm{H}_{2} \mathrm{O}_{2}$-induced HBMSCs. Figures $4 \mathrm{~A}$ and $4 \mathrm{~B}$, respectively, show ERK1/2 and Runx2 phosphorylation in HBMSCs irrespective of $\mathrm{H}_{2} \mathrm{O}_{2}$ treatment after 14 days with and without co-culture with HAMSCs. In contrast to the HBMSCs without treatment of $\mathrm{H}_{2} \mathrm{O}_{2}, \mathrm{H}_{2} \mathrm{O}_{2}$ significantly inhibited the ERK1/2 and Runx2 phosphorylation in other groups. However, when $\mathrm{H}_{2} \mathrm{O}_{2-}$ induced HBMSCs were co-cultured with HAMSCs, higher levels of phosphorylation were observed compared with $\mathrm{H}_{2} \mathrm{O}_{2-}$ induced single-culture groups and increased with the HBMSCs: HAMSCs ratio. These results suggested that HAMSCs en- hance $\mathrm{H}_{2} \mathrm{O}_{2}$-induced inhibition of ERK1/2 and Runx2 phosphorylation, which might play a role in regulating $\mathrm{H}_{2} \mathrm{O}_{2}$-induced HBMSC differentiation and mineralization.

\section{U0126 inhibited the effect of HAMSCs on $\mathrm{H}_{2} \mathrm{O}_{2}$-induced HBMSCS}

Based on the results that ERK1/2 and Runx2 phosphorylation was activated in $\mathrm{H}_{2} \mathrm{O}_{2}$-induced HBMSCs cultured with HAMSCs, we used U0126, a highly selective inhibitor of ERK1/2, to clarify whether the effect of HAMSCs was ERK1/2-dependent. An appropriate HBMSCs: HAMSCs ratio was designed based on previous results. To eliminate the influence of U0126 on HAMSCs, $\mathrm{H}_{2} \mathrm{O}_{2}$-induced HBMSCs were treated with U0126 for $24 \mathrm{~h}$ after incubation with $200 \mu \mathrm{M} \mathrm{H}_{2} \mathrm{O}_{2}$ and prior to co-culture with HAMSCs. Figures $5 \mathrm{~A}$ and $5 \mathrm{~B}$ show that HAMSCs had little effect in the downregulation of ROS generation in the presence of U0126. As expected, U0126 inhibited mineralized matrix deposition and ALP activity in $\mathrm{H}_{2} \mathrm{O}_{2}$-induced HBMSCs cocultured with HAMSCs (Figs. 6A-6C). Further, we found no significant difference in ERK1/2 and Runx2 phosphorylation between $\mathrm{H}_{2} \mathrm{O}_{2}$-induced HBMSCs with or without HAMSCs treatment consistent with the effects of U0126 (Figs. 6D and $6 \mathrm{E})$. The results suggested that by inhibiting the ERK1/2 MAPK signaling, HAMSCs failed to reverse the oxidant injury induced by $\mathrm{H}_{2} \mathrm{O}_{2}$.

\section{DISCUSSION}

Approximately $50 \%$ to $-70 \%$ of patients requiring dental implants 
$\boldsymbol{A}$

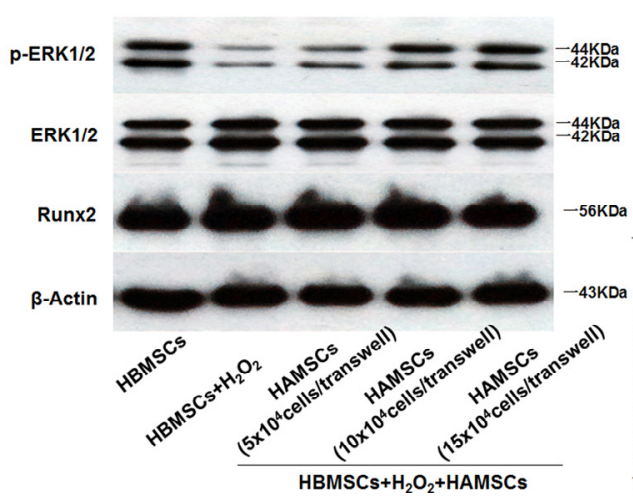

B

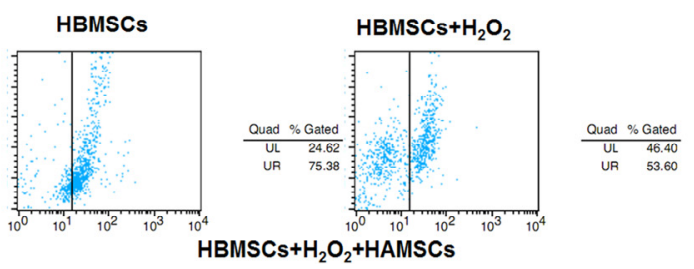

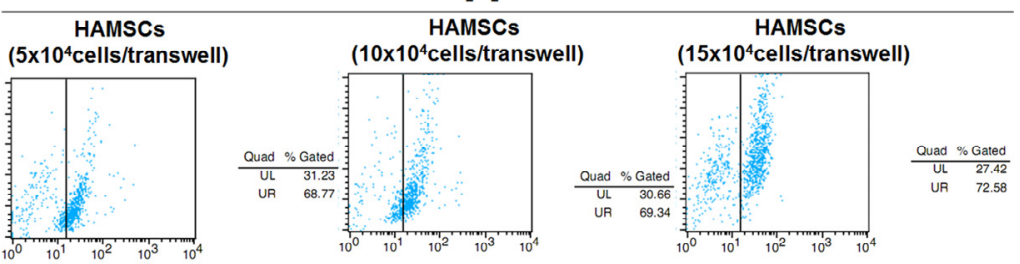

Fig. 4. Expression of $\mathrm{p}$-ERK1/2, ERK1/2, Runx2 and phospho-Runx2 proteins in $\mathrm{H}_{2} \mathrm{O}_{2}$-induced $\mathrm{HBMSC}$ cultured with or without $\mathrm{HAMSCs}$ at 14 days. (A) Protein expression of p-ERK1/2 and total ERK1/2 were determined by Western blot, $\beta$-actin served as an internal control, (B) The level of phospho-Runx2 was measured by flow cytometric analysis.

exist at various degrees of bone inadequacy clinically (Karlin, 1971; Nakano and Yatani, 2007; Stoler, 1984).A growing body of evidence suggests that increased oxidative stress may be a significant pathogenic factor of bone loss, leading to a decrease in bone formation (Baek et al., 2010; Maggio et al., 2003). Previous studies have shown that $\mathrm{H}_{2} \mathrm{O}_{2}$ causes cell injury and inhibits osteogenic differentiation in osteoblastic cells (Liu et al., 2004). In this study, an $\mathrm{H}_{2} \mathrm{O}_{2}$-induced oxidative cell injury model was built using $\mathrm{HBMSCs}$ and $200 \mu \mathrm{M} \mathrm{H}_{2} \mathrm{O}_{2}$ management for 12 h. However, pre-treatment with $\mathrm{H}_{2} \mathrm{O}_{2}$ suppressed proliferation, decreased alkaline phosphatase activity (ALP), downregulated expression of osteogenic marker protein and inhibited mineralized matrix deposition, which is similar to a previous report (Huang et al., 2015).

HAMSCs are considered a potential resource in the field of bone engineering because of their beneficial properties (Marcus et al., 2008; Tsuji et al., 2010). A previous study has suggested that HAMSCs were capable of promoting proliferation and osteogenic differentiation in HBMSCs (Wang et al., 2014), which prompted us to hypothesize the role of HAMSCs in reversing the oxidative stress-induced oxidative cell injury. First, we found that HAMSCs promoted proliferation in $\mathrm{H}_{2} \mathrm{O}_{2}-$ induced HBMSCs at 5 days, which was confirmed by flow cytometry and immunofluorescence staining of EdU, indicating that HAMSCs exerted a critical role in the prevention of $\mathrm{H}_{2} \mathrm{O}_{2-}$ derived oxidative injury. ROS generation in $\mathrm{H}_{2} \mathrm{O}_{2}$-induced HBMSCs was determined to measure the anti-oxidant properties of HAMSCs. The excessive production of ROS, such as superoxides and $\mathrm{H}_{2} \mathrm{O}_{2}$ severely damages the DNA, protein and lipids. Oxidative stress not only affects cell proliferation, but also affects differentiation (Huang et al., 2015; Lippuner, 2012; Moriwaki et al., 2014; Ollivere et al., 2012). Our findings suggest that HAMSCs inhibited ROS generation in $\mathrm{H}_{2} \mathrm{O}_{2}$-induced HBMSCs. Further investigations suggested that co-culturing
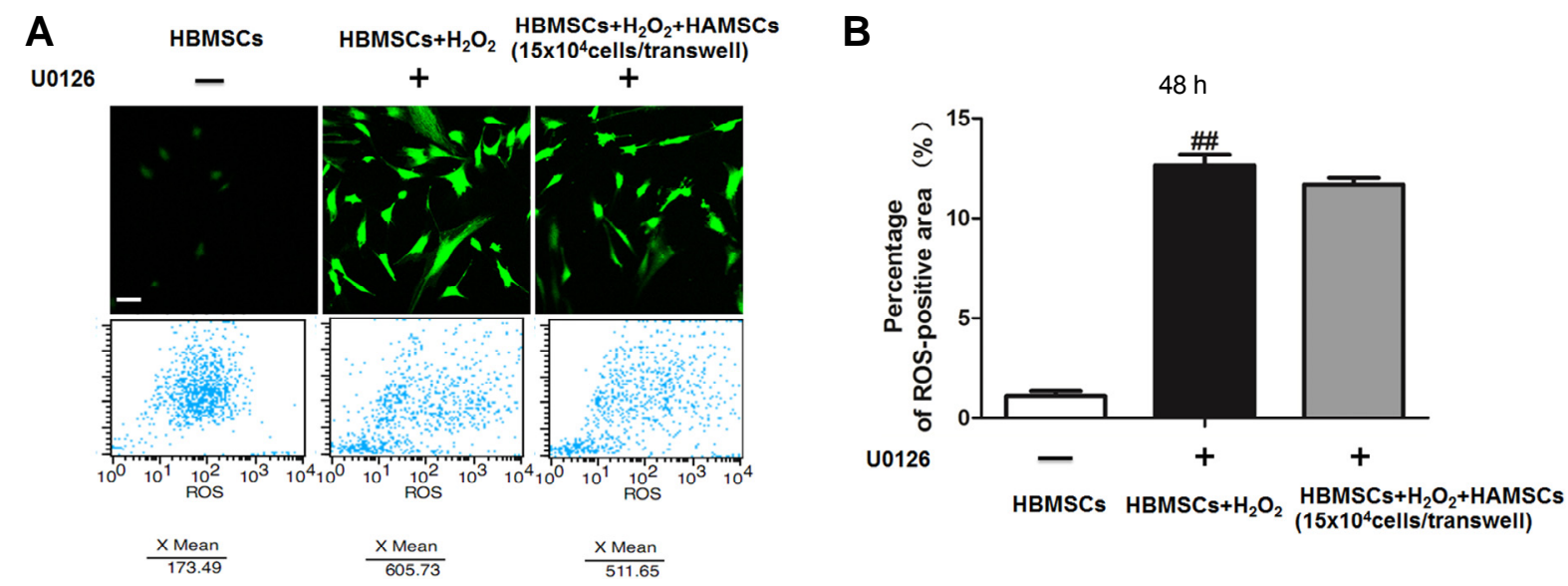

Fig. 5. U0126 inhibited the effect of HAMSCs in downregulating ROS generation. (A) Macrographs of DCFDA fluorescence were detected by fluorescence microscopy and the intensity of fluorescence was determined by flow cytometry at $48 \mathrm{~h}$, (B) Area of stained ROS-positive nodules relative to the total culture surface at $48 \mathrm{~h}$ was measured by Image-pro Plus analysis (IPP). Scale bar, $100 \mu \mathrm{m}$; \#P $<0.01$ versus the HBMSCs group. 


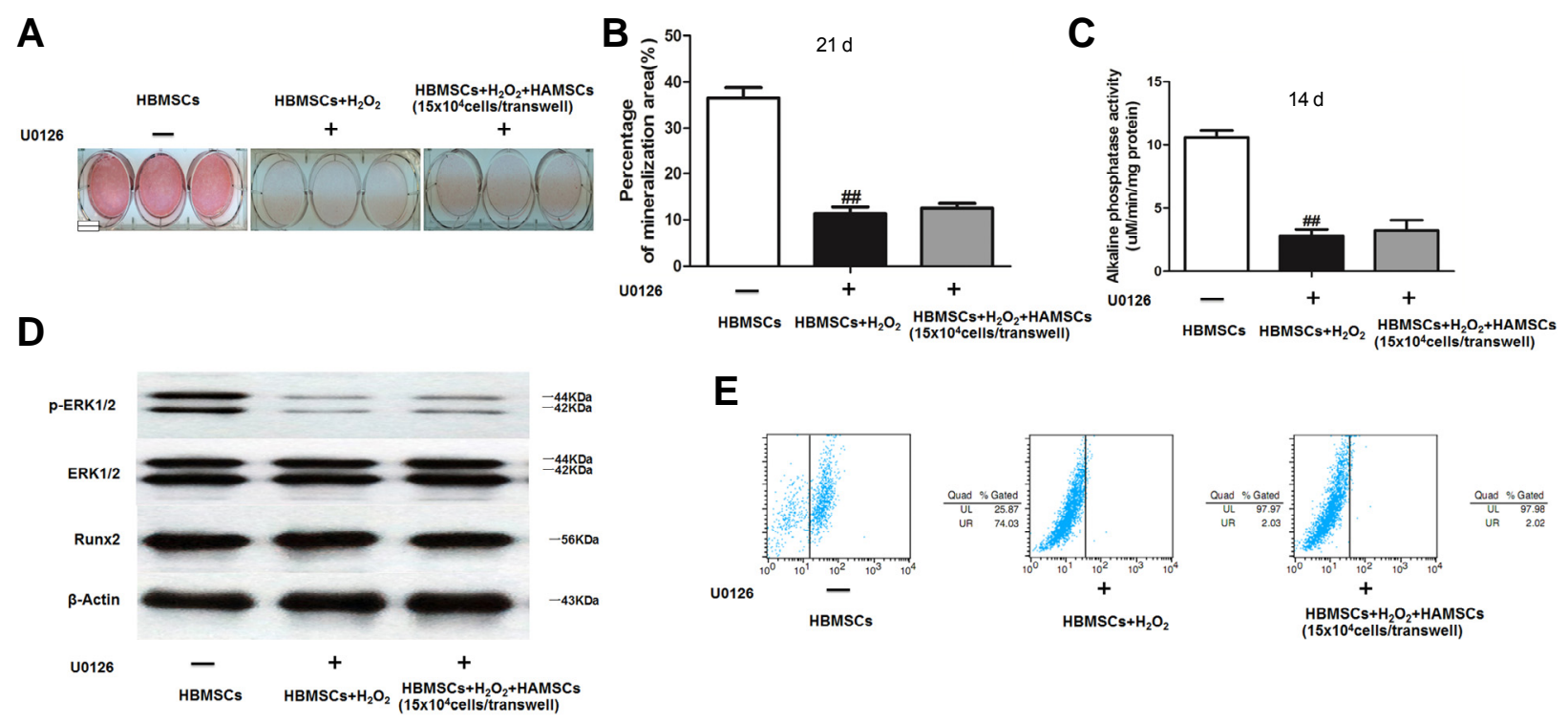

Fig. 6. U0126 inhibited the effect of HAMSCs in promoting ALP activity, mineralized matrix deposition, ERK1/2 and Runx2 phosphorylation in $\mathrm{H}_{2} \mathrm{O}_{2}$-induced HBMSCs. (A, B) Mineralized matrix deposition was measured at 21 days by Alizarin red $\mathrm{S}$ staining and Image-pro Plus analysis (IPP), (C) ALP activity was measured at 14 days using ALP assay kit, (D) Protein expression of Runx2, p-ERK1/2 and ERK1/2 was determined by Western blot at 14 days, using $\beta$-actin as an internal control, $(E)$ The level of phospho-Runx2 was measured by flow cytometric analysis at 14 days. Double bar, $12.5 \mathrm{~mm} ;{ }^{\ldots} \mathrm{P}<0.01$ versus the HBMSCs group.

with HAMSCs significantly promoted ALP activity and mineralized matrix formation in $\mathrm{H}_{2} \mathrm{O}_{2}$-induced HBMSCs. Thus, we deduced that the anti-oxidant property induced a protective effect of HAMSCs. By improving osteogenic differentiation against oxidative stress, HAMSCs might represent an appropriate therapeutic alternative against bone inadequacy.

The core purpose of our present study was to investigate the potential molecular signaling pathways engaged by HAMSCs. Signaling pathways involved in oxidative stress-induced inhibition of osteogenic differentiation consist of MAPK, Akt/mTOR/ 4EBP1, p53 and NF-kB (Martindale and Holbrook, 2002; Zeng et al., 2014). The MAPK signaling including JNK, ERK and p38 pathways have emerged as major regulators of cellular physiology and the activation of MAPK is a crucial trigger of osteogenic differentiation (Herbert et al., 2015; Hu et al., 2015; Ki et al., 2013; Qin et al., 2015). Runx2, an early-stage osteoblastic differentiation marker, typically plays an important role in osteogenesis (Franceschi et al., 2009; Phimphilai et al., 2006). Researchers believe that Runx2 overexpression enhances HBMSCs differentiation along the OB lineages, increasing new bone formation (Hu et al., 2013; Kang et al., 2013). Our study showed that HAMSCs activated ERK1/2 MAPK signaling and Runx2 in $\mathrm{H}_{2} \mathrm{O}_{2}$-induced HBMSCs, while neither the JNK nor p38 pathways appeared to be involved (data not shown). Furthermore, by using U0126, the anti-oxidant function of HAMSC was significantly downregulated by suppression of the ERK $1 / 2$ MAPK signaling pathway.

The present study highlights the antioxidant role of HAMSCs in promoting $\mathrm{H}_{2} \mathrm{O}_{2}$-induced HBMSCs proliferation and osteogenic differentiation via reduction of oxidative stress. We found that activation of the ERK1/2 MAPK signaling pathway is essential for both protective effect against oxidative stressinduced cell injury in HBMSCs and HAMSCs-mediated osteogenic effect. Suppression of ERK1/2 signaling pathways significantly inhibited the protective effect of HAMSCs against cell injury caused by oxidative stress. These data shed light on the molecular mechanism underlying the signaling cascade mediated by HAMSCs and identify the potential role of HAM$\mathrm{SCs}$ in tissue engineering.

\section{ACKNOWLEDGMENTS}

This study was supported by the National Basic Research Program of China (2012CB966902), the National Natural Science Foundation of China (81271109) and a project funded by the Priority Academic Program Development of Jiangsu Higher Education Institutions (PAPD).

\section{REFERENCES}

Baek, K.H., Oh, K.W., Lee, W.Y., Lee, S.S., Kim, M.K., Kwon, H.S., Rhee, E.J., Han, J.H., Song, K.H., Cha, B.Y., et al. (2010). Association of oxidative stress with postmenopausal osteoporosis and the effects of hydrogen peroxide on osteoclast formation in human bone marrow cell cultures. Calcif. Tissue Int. 87, 226-235.

Bai, X.C., Lu, D., Bai, J., Zheng, H., Ke, Z.Y., Li, X.M., and Luo, S.Q. (2004). Oxidative stress inhibits osteoblastic differentiation of bone cells by ERK and NF-kappaB. Biochem. Biophys. Res. Commun. 314, 197-207.

Basu, S., Michaelsson, K., Olofsson, H., Johansson, S., and Melhus, H. (2001). Association between oxidative stress and bone mineral density. Biochem. Biophys. Res. Commun. 288, 275-279.

Bourne, G.L. (1960). The microscopic anatomy of the human amnion and chorion. Am. J. Obstet. Gynecol. 79, 1070-1073.

Chen, Y.J., Chung, M.C., Jane Yao, C.C., Huang, C.H., Chang, H.H., Jeng, J.H., and Young, T.H. (2012). The effects of acellular amniotic membrane matrix on osteogenic differentiation and ERK1/2 signaling in human dental apical papilla cells. Biomaterials $33,455-463$.

Fatokun, A.A., Stone, T.W., and Smith, R.A. (2008). Responses of differentiated MC3T3-E1 osteoblast-like cells to reactive oxygen species. Eur. J. Pharmacol. 587, 35-41.

Franceschi, R.T., and Xiao, G. (2003). Regulation of the osteoblast- 
specific transcription factor, Runx2: responsiveness to multiple signal transduction pathways. J. Cell. Biochem. 88, 446-454.

Franceschi, R.T., Xiao, G., Jiang, D., Gopalakrishnan, R., Yang, S., and Reith, E. (2003). Multiple signaling pathways converge on the Cbfa1/Runx2 transcription factor to regulate osteoblast differentiation. Connec. Tissue Res. 44 Suppl 1, 109-116.

Franceschi, R.T., Ge, C., Xiao, G., Roca, H., and Jiang, D. (2009). Transcriptional regulation of osteoblasts. Cells Tissues Organs 189, 144-152.

Galli, C., Passeri, G., and Macaluso, G.M. (2010). Osteocytes and WNT: the mechanical control of bone formation. J. Dental Res. $89,331-343$.

Haigis, M.C., and Sinclair, D.A. (2010). Mammalian sirtuins: biological insights and disease relevance. Ann. Rev. Pathol. 5, 253-295

Hammond, C.L., and Schulte-Merker, S. (2009). Two populations of endochondral osteoblasts with differential sensitivity to Hedgehog signalling. Development 136, 3991-4000.

Herbert, B.A., Valerio, M.S., Gaestel, M., and Kirkwood, K.L. (2015). Sexual dimorphism in MAPK-activated protein kinase-2 (MK2) regulation of RANKL-induced osteoclastogenesis in osteoclast progenitor subpopulations. PLoS One 10, e0125387.

Hertz, J. (1956). Problems of maxillofacial and oral surgery. J. Int. College Surg. 26, 63-79.

Hu, H.M., Yang, L., Wang, Z., Liu, Y.W., Fan, J.Z., Fan, J., Liu, J., and Luo, Z.J. (2013). Overexpression of integrin a2 promotes osteogenic differentiation of hBMSCs from senile osteoporosis through the ERK pathway. Int. J. Clin. Exp. Pathol. 6, 841-852.

Hu, N., Feng, C., Jiang, Y., Miao, Q., and Liu, H. (2015). Regulative effect of Mir-205 on osteogenic differentiation of bone mesenchymal stem cells (BMSCs): possible role of SATB2/Runx2 and ERK/MAPK pathway. Int. J. Mol. Sci. 16, 10491-10506.

Huang, Q., Gao, B., Wang, L., Zhang, H.Y., Li, X.J., Shi, J., Wang, Z., Zhang, J.K., Yang, L., Luo, Z.J., et al. (2015). Ophiopogonin $D:$ A new herbal agent against osteoporosis. Bone 74, 18-28.

Ito, K., Yamada, Y., Naiki, T., and Ueda, M. (2006). Simultaneous implant placement and bone regeneration around dental implants using tissue-engineered bone with fibrin glue, mesenchymal stem cells and platelet-rich plasma. Clin. Oral. Implants Res. 17, 579-586.

Kang, Y., Kim, S., Fahrenholtz, M., Khademhosseini, A., and Yang, Y. (2013). Osteogenic and angiogenic potentials of monocultured and co-cultured human-bone-marrow-derived mesenchymal stem cells and human-umbilical-vein endothelial cells on three-dimensional porous beta-tricalcium phosphate scaffold. Acta Biomater. 9, 4906-4915.

Karlin, J.R. (1971). Oral implantology. Greater Milw. Dent. Bull. 37, 226-231.

Ki, Y.W., Park, J.H., Lee, J.E., Shin, I.C., and Koh, H.C. (2013). JNK and p38 MAPK regulate oxidative stress and the inflammatory response in chlorpyrifos-induced apoptosis. Toxicol. Lett. 218, 235-245.

Kim, S.H., Kim, K.H., Seo, B.M., Koo, K.T., Kim, T.I., Seol, Y.J., Ku, Y., Rhyu, I.C., Chung, C.P., and Lee, Y.M. (2009). Alveolar bone regeneration by transplantation of periodontal ligament stem cells and bone marrow stem cells in a canine peri-implant defect model: a pilot study. J. Periodontol. 80, 1815-1823.

Krum, S.A., Chang, J., Miranda-Carboni, G., and Wang, C.Y. (2010). Novel functions for NFkappaB: inhibition of bone formation. Nat. Rev. Rheumatol. 6, 607-611.

Leyva-Leyva, M., Barrera, L., Lopez-Camarillo, C., Arriaga-Pizano, L., Orozco-Hoyuela, G., Carrillo-Casas, E.M., Calderon-Perez, J., Lopez-Diaz, A., Hernandez-Aguilar, F., Gonzalez-Ramirez, R., et al. (2013). Characterization of mesenchymal stem cell subpopulations from human amniotic membrane with dissimilar osteoblastic potential. Stem Cells Dev. 22, 1275-1287.

Lippuner, K. (2012). The future of osteoporosis treatment - a research update. Swiss medical weekly 142, w13624.

Liu, A.L., Zhang, Z.M., Zhu, B.F., Liao, Z.H., and Liu, Z. (2004). Metallothionein protects bone marrow stromal cells against hydrogen peroxide-induced inhibition of osteoblastic differentiation. Cell Biol. Int. 28, 905-911.

Maggio, D., Barabani, M., Pierandrei, M., Polidori, M.C., Catani, M., Mecocci, P., Senin, U., Pacifici, R., and Cherubini, A. (2003). Marked decrease in plasma antioxidants in aged osteoporotic women: results of a cross-sectional study. J. Clin. Endocrinol. Metabol. 88, 1523-1527.
Maire, P. (1997). [Calibrated autologous bone grafts--their use in oral implantology. Widening--crest augmentation. Personal technic]. Rev. Stomatol. Chir. Maxillofac. 98 Suppl 1, 27-30.

Marcus, A.J., Coyne, T.M., Rauch, J., Woodbury, D., and Black, I.B. (2008). Isolation, characterization, and differentiation of stem cells derived from the rat amniotic membrane. Differentiation 76 130-144.

Martindale, J.L., and Holbrook, N.J. (2002). Cellular response to oxidative stress: signaling for suicide and survival. J. Cell. Physiol. 192, 1-15.

Mody, N., Parhami, F., Sarafian, T.A., and Demer, L.L. (2001). Oxidative stress modulates osteoblastic differentiation of vascular and bone cells. Free Radic. Biol. Med. 31, 509-519.

Moriwaki, S., Suzuki, K., Muramatsu, M., Nomura, A., Inoue, F., Into, T., Yoshiko, Y., and Niida, S. (2014). Delphinidin, one of the major anthocyanidins, prevents bone loss through the inhibition of excessive osteoclastogenesis in osteoporosis model mice. PLoS One 9, e97177.

Muthusami, S., Ramachandran, I., Muthusamy, B., Vasudevan, G., Prabhu, V., Subramaniam, V., Jagadeesan, A., and Narasimhan, S. (2005). Ovariectomy induces oxidative stress and impairs bone antioxidant system in adult rats. Clin. Chim. Acta 360, 81 86

Nakano, T., and Yatani, H. (2007). [Bone augmentation of dental implant treatment]. Clin. Calcium 17, 256-262.

Ollivere, B., Wimhurst, J.A., Clark, I.M., and Donell, S.T. (2012). Current concepts in osteolysis. J. Bone Joint Surg. Br. 94, 10-15

Ozeki, K., Aoki, H., and Fukui, Y. (2008). The effect of adsorbed vitamin $\mathrm{D}$ and $\mathrm{K}$ to hydroxyapatite on ALP activity of MC3T3-E1 cell. J. Mater. Sci. 19, 1753-1757.

Ozgocmen, S., Kaya, H., Fadillioglu, E., Aydogan, R., and Yilmaz, Z (2007). Role of antioxidant systems, lipid peroxidation, and nitric oxide in postmenopausal osteoporosis. Mol. Cell. Biochem. 295, 45-52.

Phimphilai, M., Zhao, Z., Boules, H., Roca, H., and Franceschi, R.T. (2006). BMP signaling is required for RUNX2-dependent induction of the osteoblast phenotype. J. Bone Miner. Res. 21, 637646.

Qin, L., Tang, B., Deng, B., Mohan, C., Wu, T., and Peng, A. (2015). Extracellular regulated protein kinases play a key role via bone morphogenetic protein 4 in high phosphate-induced endothelial cell apoptosis. Life Sci. 131, 37-43.

Reinholz, G.G., Getz, B., Pederson, L., Sanders, E.S., Subramaniam, M., Ingle, J.N., and Spelsberg, T.C. (2000). Bisphosphonates directly regulate cell proliferation, differentiation, and gene expression in human osteoblasts. Cancer Res. 60, 6001-6007.

Salasznyk, R.M., Klees, R.F., Hughlock, M.K., and Plopper, G.E. (2004). ERK signaling pathways regulate the osteogenic differentiation of human mesenchymal stem cells on collagen I and vitronectin. Cell Commun. Adhes. 11, 137-153.

Schindeler, A., and Little, D.G. (2006). Ras-MAPK signaling in osteogenic differentiation: friend or foe? J. Bone Miner Res. 21 1331-1338.

Schneider, G.B., Whitson, S.W., and Cooper, L.F. (1999). Restricted and coordinated expression of beta3-integrin and bone sialoprotein during cultured osteoblast differentiation. Bone 24, 321-327.

Sendur, O.F., Turan, Y., Tastaban, E., and Serter, M. (2009). Antioxidant status in patients with osteoporosis: a controlled study. Joint Bone Spine 76, 514-518.

Simmons, C.A., Matlis, S., Thornton, A.J., Chen, S., Wang, C.Y., and Mooney, D.J. (2003). Cyclic strain enhances matrix mineralization by adult human mesenchymal stem cells via the extracellular signal-regulated kinase (ERK1/2) signaling pathway. J. Biomech. 36, 1087-1096.

Sontakke, A.N., and Tare, R.S. (2002). A duality in the roles of reactive oxygen species with respect to bone metabolism. Clin. Chim. Acta $318,145-148$

Stoler, A. (1984). Oral implantology today. Florida Dental J. 55, 3637,54 .

Tsuji, H., Miyoshi, S., Ikegami, Y., Hida, N., Asada, H., Togashi, I., Suzuki, J., Satake, M., Nakamizo, H., Tanaka, M., et al. (2010) Xenografted human amniotic membrane-derived mesenchymal stem cells are immunologically tolerated and transdifferentiated into cardiomyocytes. Circ. Res. 106, 1613-1623.

Wang, S., Zhang, Z., Zhao, J., Zhang, X., Sun, X., Xia, L., Chang, Q., Ye, D., and Jiang, X. (2009). Vertical alveolar ridge augmen- 
tation with beta-tricalcium phosphate and autologous osteoblasts in canine mandible. Biomaterials 30, 2489-2498.

Wang, W. Olson, D., Cheng, B., Guo, X and Wang, K. (2012) Sanguis Draconis resin stimulates osteoblast alkaline phosphatase activity and mineralization in MC3T3-E1 cells. J. Ethnopharmacol. 142, 168-174.

Wang, Y., Yan, M., Yu, Y., Wu, J., Yu, J., and Fan, Z. (2013). Estrogen deficiency inhibits the odonto/osteogenic differentiation of dental pulp stem cells via activation of the NF-kappaB pathway. Cell Tissue Res. 352, 551-559.

Wang, Y., Yin, Y., Jiang, F., and Chen, N. (2014). Human amnion mesenchymal stem cells promote proliferation and osteogenic differentiation in human bone marrow mesenchymal stem cells. J. Mol. Histol. 46, 13-20.

Wang, Y., Yin, Y., Jiang, F., and Chen, N. (2015). Human amnion mesenchymal stem cells promote proliferation and osteogenic differentiation in human bone marrow mesenchymal stem cells. J. Mol. Histol. 46, 13-20.

Witko-Sarsat, V., Friedlander, M., Capeillere-Blandin, C., NguyenKhoa, T., Nguyen, A.T., Zingraff, J., Jungers, P., and DescampsLatscha, B. (1996). Advanced oxidation protein products as a novel marker of oxidative stress in uremia. Kidney Int. 49, 13041313.

Xia, Z., Dickens, M., Raingeaud, J., Davis, R.J., and Greenberg,
M.E. (1995). Opposing effects of ERK and JNK-p38 MAP kinases on apoptosis. Science 270, 1326-1331.

Xiao, G., Gopalakrishnan, R., Jiang, D., Reith, E., Benson, M.D. and Franceschi, R.T. (2002). Bone morphogenetic proteins, extracellular matrix, and mitogen-activated protein kinase signaling pathways are required for osteoblast-specific gene expression and differentiation in MC3T3-E1 cells. J. Bone Miner. Res. 17, 101-110.

Yang, S., Madyastha, P., Bingel, S., Ries, W., and Key, L. (2001). A new superoxide-generating oxidase in murine osteoclasts. J. Biol. Chem. 276, 5452-5458.

Zeng, X., Tian, J., Cai, K., Wu, X., Wang, Y., Zheng, Y., Su, Y., and Cui, L. (2014). Promoting osteoblast differentiation by the flavanes from Huangshan Maofeng tea is linked to a reduction of oxidative stress. Phytomedicine 21, 217-224.

Zhang, W., and Liu, H.T. (2002). MAPK signal pathways in the regulation of cell proliferation in mammalian cells. Cell Res. 12, 9-18.

Zhang, D., Jiang, M., and Miao, D. (2011). Transplanted human amniotic membrane-derived mesenchymal stem cells ameliorate carbon tetrachloride-induced liver cirrhosis in mouse. PLoS One 6, e16789.

Zhao, J., Zhang, Z., Wang, S., Sun, X., Zhang, X., Chen, J., Kaplan, D.L., and Jiang, X. (2009). Apatite-coated silk fibroin scaffolds to healing mandibular border defects in canines. Bone 45, 517-527. 